\title{
Corrigendum: The genome sequence of the orchid Phalaenopsis equestris
}

Jing Cai, Xin Liu, Kevin Vanneste, Sebastian Proost, Wen-Chieh Tsai, Ke-Wei Liu, Li-Jun Chen, Ying He, Qing Xu, Chao Bian, Zhijun Zheng, Fengming Sun, Weiqing Liu, Yu-Yun Hsiao, Zhao-Jun Pan, Chia-Chi Hsu, Ya-Ping Yang, Yi-Chin Hsu, Yu-Chen Chuang, Anne Dievart, Jean-Francois Dufayard, Xun Xu, Jun-Yi Wang, Jun Wang, Xin-Ju Xiao, Xue-Min Zhao, Rong Du, Guo-Qiang Zhang, Meina Wang, Yong-Yu Su, Gao-Chang Xie, Guo-Hui Liu, Li-Qiang Li, Lai-Qiang Huang, Yi-Bo Luo, Hong-Hwa Chen, Yves Van de Peer \& Zhong-Jian Liu Nat. Genet. 47, 65-72 (2015); published online 24 November 2014; corrected after print 9 January 2015; corrected after print 6 February 2015

In the version of this article initially published, the legend for Figure $1 \mathrm{~b}$ referred to red arrows indicating the inferred divergence dates. No arrows are depicted in the figure, so this sentence has been removed from the figure legend in the HTML and PDF versions of the article.

\section{Corrigendum: Mutations in the voltage-gated potassium channel gene KCNH1 cause Temple-Baraitser syndrome and epilepsy}

Cas Simons, Lachlan D Rash, Joanna Crawford, Linlin Ma, Ben Cristofori-Armstrong, David Miller, Kelin Ru, Gregory J Baillie, Yasemin Alanay, Adeline Jacquinet, François-Guillaume Debray, Alain Verloes, Joseph Shen, Gözde Yesil, Serhat Guler, Adnan Yuksel, John G Cleary, Sean M Grimmond, Julie McGaughran, Glenn F King, Michael T Gabbett \& Ryan J Taft Nat. Genet. 47, 73-77 (2015); published online 24 November 2014; corrected after print 6 February 2015

In the version of this article initially published, in Figure 2a, the order of the protein alterations for variants c. $1465 \mathrm{C}>\mathrm{T}$ and c.1480A $>\mathrm{G}$ was inverted. The correct protein alterations for these two variants are p.Leu489Phe and p.Ile494Val, respectively. This error has been corrected in the HTML and PDF versions of the article. 